\title{
Hacia una escuela violeta: la formación inicial del alumnado de Educación Primaria en coeducación
}

'Towards a violet school: teachers' of Primary education initial training in coeducation

\author{
Begoña Sánchez Torrejón \\ e-mail: begonia.sanchez@uca.es \\ Universidad de Cádiz. España
}

Zulema Barea Villalba

e-mail: zulemabarea@hotmail.com

Universidad de Cádiæ: España

\section{Resumen}

La presente investigación surge del objetivo de detectar la formación inicial en materia de coeducación que posee el alumnado del Grado de Educación Primaria de la Universidad de Cádiz, futuras maestras y futuros maestros. La importancia de la formación inicial del profesorado de la etapa de Educación Primaria es un elemento clave en la construcción de una escuela libre de sexismo y, por ende, de una sociedad democrática que propicie la igualdad entre hombres y mujeres. Hemos utilizado la metodología cuantitativa, concretamente el instrumento planteado para recabar la información ha sido el cuestionario. La muestra del universo poblacional está compuesta por el alumnado de los tres cursos del cuarto año del Grado de Educación Primaria de la Universidad de Cádiz, un total de 195 alumnas y alumnos. Los resultados más destacados demuestran que el alumnado posee escasa formación en coeducación y, por tanto, la incapacidad de trabajar en un futuro la igualdad de oportunidades en los centros escolares entre las niñas y los niños, demandando la necesidad de formación en esta temática para poder implementar en un futuro la coeducación en sus aulas. Finalizamos con el planteamiento de propuestas de intervención prácticas para favorecer el camino de la coeducación en nuestras escuelas mediante la formación inicial del profesorado.

Palabras clave: coeducación; formación inicial del profesorado; Educación Primaria; igualdad de oportunidades; género

\begin{abstract}
The present research arises from the objective of detecting the initial formation in matters of coeducation that has the students of the Degree of Primary Education of University of Cadiz; future teachers. The importance of the initial training of the teachers of the Primary Education is a key element in the construction of a school free of sexism and therefore, of a democratic society that promotes equality between men and women. We have used the quantitative methodology, specifically the instrument to gather information has been the questionnaire. The population sample is composed of the students of the three courses of the fourth year of the Primary Education Degree of the University of Cadiz, a total of 195 students. The most outstanding results show that the students have little training in coeducation and therefore the inability to work in the future the equal opportunities between girls and boys in schools, while demanding the need of training in this subject to be able to implement coeducation in their classrooms. We finish with an approach to practical intervention proposals to favor the way of coeducation in our schools through the initial teacher training.
\end{abstract}

Keywords: coeducation; initial teacher training; Primary Education; equal opportunities; gender

Recibido / Received: 15-01-2019

Aceptado / Accepted: 10-04-2019

Publicación en línea / Published online: 01-07-2019

Cómo referenciar este artículo / How to reference this article:

Sánchez Torrejón, B., \& Barea Villalba, Z. (2019). Hacia una escuela violeta: la formación inicial del alumnado de Educación Primaria en coeducación. Tendencias Pedagógicas, 34, pp. 76-92. doi: 10.15366/tp2019.34.007 


\section{Introducción}

El trabajo de investigación que presentamos a continuación parte de la necesidad de construir una escuela libre de sexismo, que favorezca la igualdad de oportunidades en los centros escolares entre las niñas y los niños. Esta investigación nace de la urgente demanda de formación inicial en los planes de formación del profesorado de Educación Primaria en materia de coeducación como elemento transformador para deconstruir el sexismo y las desigualdades de género en los centros escolares. Conocer la formación y las actitudes del futuro profesorado de Educación Primaria sobre la coeducación, como señala Scraton (1992), nos permitirá evaluar y establecer nuevas propuestas de formación que sean apropiadas a los déficits del profesorado, y propiciar una nueva cultura de género más igualitaria.

El profesorado es uno de los agentes claves en la creación de una cultura de género, libre de estereotipos sexistas, protagonistas en el cambio de la desigualdad entre hombres y mujeres en las aulas, para poder construir una sociedad democrática en donde estén presentes en igualdad hombres y mujeres. Defendemos la importancia de la formación inicial del profesorado para la supresión de las discriminaciones y estereotipos sexistas en nuestro sistema educativo. Una de las metas que plantea Martín Vico (2007) para poder alcanzar la coeducación en la escuela, es la necesidad de que cada docente debe tener las competencias necesarias para coeducar, mediante la formación inicial basada en la igualdad de oportunidades entre mujeres y hombres.

Como postula Antón (2007) educar es una tarea basada en las relaciones humanas, la cual no sólo abarca los conocimientos y técnicas del profesional, sino que está impregnada de sentimientos y emociones, tanto positivas como negativas. El profesorado tiene un papel fundamental, ya que es un agente socializador imprescindible que se encarga de trabajar para el desarrollo y progreso de una ciudadanía libre de prejuicios sexistas. La escuela tiene la obligación de ser compensadora de las desigualdades de género, transformadora de una realidad que todavía arrastra la herencia social en la que se mantiene el papel subsidiario para las mujeres.

Suberviola (2012), denuncia que siguen existiendo marcados estereotipos sexistas transmitidos por el profesorado y aún mucha confusión sobre la educación mixta como herramienta suficiente para la consecución de la igualdad entre hombres y mujeres. Weiner (2000) critica la ausencia de la perspectiva de género en la formación del profesorado en el marco de la Unión Europea y puntualiza cómo siendo el ámbito educativo, caracterizado por una gran presencia de mujeres, se sigue perpetuando las desigualdades de género entre hombres y mujeres. De este modo, como apunta Ballarín «la escuela contribuye a reproducir desigualdades, manteniendo de forma sutil los roles sexistas, ignorando los valores y las experiencias de las mujeres» (2001, p.137). El Instituto de la Mujer en 2004, denunciaba como la formación del profesorado tanto en lo referido a la formación inicial como a la formación permanente sobre las desigualdades de las mujeres y desde una perspectiva de género, ha sido una asignatura pendiente en nuestro país, entre una de las causas de este problema apunta a la invisibilidad del propio profesorado en ejercicio.

Es fundamental subrayar que entre las competencias del futuro profesorado en Educación Primaria estén contempladas competencias relacionadas con la igualdad de género, teniendo en cuenta el momento de adaptación de muchas universidades al marco Europeo en la construcción del Espacio Europeo de Educación Superior (EEES). Reflejado también en la Ley de Igualdad entre Mujeres y Hombres de 2007 y con una directriz clara en la guía realizada por la Agencia Nacional de Evaluación de la Calidad y la Acreditación (ANECA). Estos esfuerzos institucionales nos llevan a reflexionar e integrar estos contenidos en los futuros planes de estudio dentro de los marcos de enseñanza europeos.

La coeducación se basa en el principio de igualdad entre hombres y mujeres como defiende Ballarín (2001), no podemos olvidar que partimos de un marco legislativo sobre igualdad, que debemos tener presente y materializar en nuestros centros educativos. Esta se ve también reflejada en la reglamentación básica de los planes de estudio universitarios, la cual refleja indicaciones específicas sobre la inclusión de la perspectiva de género en la formación básica del alumnado. Consideramos de urgente necesidad la puesta en práctica de mecanismos efectivos para formar al profesorado desde su formación inicial en un análisis crítico con perspectiva de género, favoreciendo la reflexión epistemológica del carácter androcéntrico de la construcción del discurso hegemónico imperante, basados en metodologías innovadoras, para posteriormente educar en igualdad de género desde edades tempranas al alumnado de Educación Primaria y así favorecer la formación de ciudadanas y ciudadanos libres de sexismo en espacios democráticos. 


\section{Arquitectura conceptual}

El fin que tiene esta revisión teórica es situar el problema dentro del conjunto de conocimientos que permita orientar nuestra búsqueda y ofrecer una conceptualización adecuada. En la estructura conceptual de esta investigación comenzaremos con un breve recorrido histórico de la coeducación en España y revisión del concepto de coeducación a lo largo de la historia, hasta la actualidad. Estos aspectos facilitan una base teórica para conocer y comprender todo lo que está relacionado con la coeducación.

Desde estos referentes conceptuales y, en segundo lugar, nos centraremos en la formación inicial del profesorado de Educación Primaria en coeducación, profundizaremos en reflejar la estrecha relación entre la temática de objeto de estudio de la presente investigación y la formación de los futuros docentes, elemento clave para propiciar una escuela libre de sexismo y desigualdad.

\subsection{El camino recorrido de la coeducación}

Nos centraremos a continuación en un breve recorrido histórico de la coeducación en España, hasta llegar a nuestros días, desde los diversos momentos históricos que las han abordado, el concepto de coeducación ha ido evolucionando a lo largo de los años. La evolución del concepto de coeducación como señala Subirats (1994) ha sido simultáneo con el cambio de posiciones de las mujeres en la sociedad. El modelo escolar segregado, ha subsistido en nuestro país hasta los 1970 y con él la idea de dos naturalezas distintas que determinan dos funciones sociales diferentes.

Comenzaremos este largo y arduo camino recorrido en el siglo XVIII, en este periodo las mujeres no tenían derecho a la educación, bajo el discurso misógino sobre la inferioridad física e intelectual de las mujeres como señala Scanlon (1986), se argumentaba su exclusión al derecho de ciudadanía y por tanto de la educación. Relegándolas al aprendizaje de sus tradicionales papeles vinculados al cuidado de la familia y a las labores domésticas.

En España, en el siglo XIX desde la constitución de las Cortes de Cádiz en 1812, las mujeres fueron anuladas en el discurso liberal, invisibilizadas en el discurso de la ciudadanía, pero sí ensalzadas como madres, confinadas en el espacio privado (Sohn, 2002). Las Cortes de Cádiz promulgaban que la educación sería una enseñanza universal, uniforme y obligatoria, pero dentro de esa universalidad se olvidaron incluir a las mujeres. En la primera legislación del 1821, «El Informe Quintana», regulaba tres niveles de educación, pero el alumnado femenino quedaba relegado a la educación doméstica, limitada a las funciones del hogar.

Con la llegada de la Ley Moyano de 1857, la obligatoriedad de la enseñanza se extiende también a las niñas y a los niños de entre 6 y 9 años. Eso sí, las materias eran diferentes para cada sexo, donde la moralidad era muy importante, las escuelas estaban segregadas, las posibilidades de continuar la educación más allá de la enseñanza elemental se complicaba para las niñas. A excepción de las escuelas de formación de maestras (Ballarín, 2001).

A nadie se le ocurrió pensar que las mujeres solicitarían una educación secundaria o universitaria, y como la ley no lo prohibía, las mujeres comenzaron a solicitarlo. En 1868, las primeras mujeres se matriculan en los institutos de secundaria. En 1888 la posición quedó clara: las mujeres para poder estudiar en la universidad necesitaban unas serie de permisos: del padre, del rector, etc. Hasta 1910 no se permite el libre acceso de la mujer a la universidad, aunque no podrán ejercer determinados oficios (Flecha, 1989).

Es interesante destacar las aportaciones a principios del siglo XX, entre 1901 y 1906, la Escuela Moderna de Ferrer i Guàrdia, junto a la experiencia de la Institución Libre de Enseñanza, serán el antecedente inmediato de la organización del sistema escolar bajo la Segunda República y el gobierno autónomo de la Generalidad de Cataluña, estructurado bajo el modelo de la escuela mixta para ambos sexos, extendió su influencia sobre treinta y cuatro centros escolares.

La República trajo notables cambios y avances para las mujeres a todos los niveles, no solo fue el acceso al voto femenino, fueron grandes los progresos para estas en el ámbito jurídico, social, laboral y educativo. En 1930 el nivel de analfabetismo en España era muy alto, llegándose a reducir hasta un $45 \%$ y se plantea una escuela laica, incorporando la coeducación en las aulas. Uno de los grandes avances educativos fue entre 1935 y 1936 al duplicarse el acceso a la educación superior. Mediante el 
Decreto de 28 de agosto de 1931, se estableció la coeducación en todos los centros oficiales de segunda enseñanza y, por lo tanto, los institutos femeninos existentes debieron proceder a la admisión de matrículas de ambos sexos.

Aunque como apunta Ballarín (2001) las experiencias de escuela mixta fueron minoritarias, pues sólo una minoría de centros llegó a tener carácter mixto, incluso en la etapa de la Segunda República cuando la coeducación fue admitida y considerada necesaria. Pero este corto período de la implantación de la escuela mixta desde la República fue muy beneficioso para todas las niñas, ya que aumentaron notablemente las tasas de su escolarización y pudieron ampliar su ámbito de actuación.

En 1936 de la Guerra Civil, y su triunfo en 1939, se instauró la dictadura del General Francisco Franco. Sin embargo, el régimen franquista que se instala en España durante cuarenta años a partir de 1939 significó un claro retroceso y la vuelta a una concepción de mujer vinculada exclusivamente al mundo privado. De esta forma, desde el verano de 1936 y de manera reiterada a lo largo de las siguientes décadas, el gobierno según Puelle (1991), derogará la coeducación a través de una sucesión de órdenes encaminadas a afianzar una clara diferencia social entre hombres y mujeres. Al final de la guerra civil queda cerrada, por un largo período, la opción de la escuela mixta.

En el período de dictadura, el gobierno impondrá una sucesión de órdenes encaminadas a afianzar una clara diferencia social entre hombres y mujeres como las que se refieren a la derogación de la coeducación y en consecuencia, la promulgación de una escuela segregada (Escolano, 2002). La etapa franquista significa la diferenciación de currículums y de la escuela separada para las mujeres, promoviendo de esta manera la desvalorización profesional de su formación escolar. La Sección Femenina de la Falange, será la encargada de la educación de las niñas, esta se propondrá difundir un modelo pedagógico dirigido a inculcar a la mujer que la finalidad de su educación se circunscribía a los límites de su función de madre y responsable del hogar, fomentando la figura del «ángel del hogar».

Las mujeres quedarían confinadas de nuevo en el ámbito de lo doméstico, negándoles la posibilidad de desarrollar su nivel cultural, derecho que habían conquistado en el primer tercio del siglo. En definitiva, se produce el regreso a los principios desiguales ya formulados en el siglo XVIII sobre la educación de las niñas.

Con la llegada de la democracia en España, tras el fin de la dictadura franquista, en los años 70, la necesidad de readaptación y renovación del sistema escolar era una prioridad. En 1970 daría lugar la Ley General de Educación que derogaba la obsoleta Ley Moyano y que regulaba la implantación de la escuela mixta y se abrió la puerta a la educación conjunta de las niñas y los niños, la cual marcó el camino a la escuela mixta. Esta impulsó la igualdad de oportunidades y el mismo currículum para ambos sexos.

La Ley Orgánica de Ordenación General del Sistema Educativo (LOGSE), entró en vigor en 1990, amplió la enseñanza obligatoria hasta los 16 años y procuró introducir la igualdad en todos los aspectos de la vida escolar. Esta ley fijó la importancia de la educación igualitaria, otorgándole especial énfasis a la igualdad en todos los aspectos y niveles de la educación española.

Subirats y Brullet (1988), denuncian que frente a la aparente igualdad que plantea la educación mixta, aún subsisten elementos discriminatorios que seguían moldeando a las mujeres como seres pasivos y dependientes y a los varones como agresivos y dominantes. Entre los diversos aspectos discriminatorios que las autoras evidencian, nos encontramos con el sexismo en el lenguaje, el androcentrismo científico, la trasmisión de éstos a través de los libros de texto, así como la discriminación en el trato hacia unos y hacia otras y la propia desigualdad en la organización escolar, elementos desiguales que en la actualidad perpetúan el sexismo en los centros escolares.

A lo largo del presente recorrido histórico hemos comprobado que los hombres y las mujeres siempre han estado bajo la influencia de determinados roles de género desiguales, decretando las cualidades y capacidades de cada uno y de cada una. El proceso de lucha social y política de las mujeres en la consecución de sus derechos a lo largo de la edad contemporánea en España, ha sido duro y lleno de grandes obstáculos, que tuvieron que superar para llegar a ser consideradas como ciudadanas de pleno derecho. El lento acceso de las mujeres a la educación, que se refleja en la historia de la educación de las mujeres en España como señala Ballarín (2001) es hasta nuestra actualidad una conquista educativa que debemos conseguir.

Estas desigualdades sexistas se trasladaban al ámbito educativo, en el cual hasta finales del siglo XX las mujeres y los hombres de nuestro país eran instruidas e instruidos de forma diferenciada, posicionando a los hombres en un estatus superior que a las mujeres. Este hecho nos deja como 
herencia una sociedad patriarcal, en el que en la actualidad aún observamos que en los temarios escolares se siguen transmitiendo una cultura androcéntrica. Los avances legislativos que nos amparan y la lucha de los movimientos feministas, han sido factores determinantes en el camino hacia la igualdad, pero como hemos indicado anteriormente los resquicios de esa sociedad sexista siguen conviviendo en nuestra sociedad y por tanto en la escuela.

Atendiendo a la definición planteada por Monasterio, González y García (2011) entendemos que la coeducación es un proceso que nos guía hacia las diversidades, reconociendo las diferencias de cada uno y cada una y otorgándole el valor necesario para educar en la equidad de derechos. Por lo tanto, la tarea de coeducar tiene su base en la búsqueda de la igualdad entre hombres y mujeres, educando en valores como la igualdad, el respeto y la tolerancia. Estas mismas autoras defienden la coeducación como una metodología transversal, es decir, un contenido que debe estar presente en todos los ámbitos de la educación, tratándose como un proceso continuo y no como un hecho puntual.

Este aspecto resulta muy llamativo ya que, hoy en día muchas y muchos docentes por el hecho de conmemorar un día significativo del calendario afirman trabajar la coeducación en las aulas, mientras tanto día a día va alejándose de esta realidad mediante un lenguaje sexista, estereotipos marcados en su curriculum oculto.

Cuando hablamos de coeducación no podemos olvidar que nos referimos tanto a hombres como mujeres:

«Coeducar es educar fuera del modelo dominante, el simbólico patriarcal (...); y eso significa que tanto las mujeres como los hombres hemos de pensar de nuevo, desde otros referentes, qué significa ser una mujer, ser un hombre, en el contexto histórico en el que vivimos.» (Blanco, 2007, p. 24)

Del mismo modo, Cabeza (2010) se refiere al término coeducar como el desarrollo integral de todo el alumnado, prestando especial atención al conocimiento del otro sexo y al enriquecimiento mutuo de ambos sexos. Centrado en corregir los estereotipos sexistas, propone un currículum equilibrado que elimine los sesgos sexistas y desarrollar todas las capacidades individuales con independencia del género.

\subsection{La formación inicial del profesorado de Educación Primaria en coeducación}

Seguidamente, ahondaremos en el papel activo del profesorado como uno de los componentes de la comunidad educativa más importantes en la construcción de la cultura de género en la escuela para combatir las desigualdades de género en las aulas. El profesorado trasmite en su acción educativa siguiendo a Colás (2004), no sólo el conocimiento, además la forma de ver e interpretar el mundo al alumnado; por ello, una adecuada y actualizada formación en igualdad de género es clave para avanzar hacia la coeducación. Diferentes autores García, Salas, Rodríguez y Sabuco (2013), denuncian la escasa atención que se le presta a este tema desde la práctica de la formación inicial docente.

Partimos de la idea que ser docente es una profesión que demanda no sólo conocimientos, ni procedimientos profesionales, también determinas actitudes personales y profesionales. Para poder emprender el camino de la coeducación es necesario tener valores de igualdad, pero no podemos obviar el currículum oculto que el profesorado posee: «Conjunto de normas y valores inconscientes de conducta, aprendidas en la primera infancia y perpetuadas a través de los contenidos y sobre todo a través de los comportamientos (...) del profesorado respecto a alumnos y alumnas» (Altable, 1993, p. 130).

Como defiende Bromme (1988), estos conocimientos tácitos, saberes implícitos del profesorado, aunque el propio profesorado no se haya percatado de poseerlos son la base de sus actuaciones educativas. Las ideas y las diversas creencias sobre los papeles masculinos y femeninos constituyen un bagaje sobre la conceptualización sobre la diferencia sexual. Estos conocimientos personales, generados a través de las propias experiencias que cada individuo, ha tenido en función de su sexo, la familia, la escuela, o en otros entornos de socialización son claves en la perpetuación o eliminación de estereotipos y roles sexistas.

Somos conscientes que la actitud del profesorado es fundamental en la reproducción o en la eliminación de estereotipos sexistas. Como señala Fernández (1996) a través de sus interacciones y sus discursos, el profesorado puede reproducir y perpetuar el sexismo en su aula o por el contrario, proponer acciones enfocadas a una educación para la igualdad de oportunidades. No podemos olvidar 
las palabras de Santos Guerra «El educador trabaja con unos "materiales" de altísima delicadeza e importancia: emociones, sentimientos, concepciones, actitudes, valores, ideas (...) Son "materiales" que no obedecen a las leyes» (2008, p.17).

La formación inicial del profesorado de Educación Primaria en coeducación, debe favorecer la reflexión sobre los propios prejuicios y debe aportar las claves para leer la propia realidad educativa desde una perspectiva no sexista. Aunque durante los últimos años el profesorado ha emprendido diversos proyectos en los centros escolares que fomentan la coeducación, la mayoría del profesorado no se cuestiona la cultura androcéntrica escolar limitándose a reproducir las desigualdades que han recibido.

Bolaños (2007) y Colás (2004) abordan desde tres niveles la importancia de afrontar en los procesos de formación del profesorado en los que se manifiestan las relaciones género-poder: nivel macroestructural, nivel mesoestructural y nivel microestructural.

- El nivel macroestructural: hace referencia al imaginario social sobre género y poder. Este aspecto incluye las representaciones establecidas a nivel cultural y social.

- El nivel mesoestructural: pone en relación el binomio género-poder con la realidad próxima y los contextos de interacción. Hace alusión a las representaciones sociales de género en los contextos próximos de socialización, tales como familia, escuela o escenarios profesionales. En este nivel el profesorado en formación debería identificar marcas de género-poder patriarcales en la cultura escolar.

- El nivel microestructural: hace referencia a la dimensión más personal e interna, que se manifiesta y expresa en la interacción con otras personas. Se refiere a la experiencia personal del profesorado en formación.

El RD 861/2010 de 2 de julio, que completa y modifica el RD 1393/2007 de 29 de octubre, establece que los Grados relacionados con la educación deberían incluir contenidos formativos relacionados con la igualdad o la prevención de la violencia, pero desgraciadamente aún en la mayoría de las titulaciones universitarias de formación del profesorado no se incluyeron estos aspectos.

Una investigación realizada por Rebollo (2013), sobre opiniones y actitudes del profesorado en relación con la igualdad, señala que aproximadamente un $35 \%$ del profesorado masculino y un $25 \%$ del femenino no reconoce ni percibe prácticas de discriminación y desigualdad en la escuela, mostrando su mayor dificultad perceptiva en el plano de la práctica educativa.

Por todo ello, como apunta Bolaños «el profesorado reconozca la igualdad de niñas y niños, comprenda las realidades que vivencian y reconozca las concepciones de género y poder que manejan. Además, debe contribuir a la formación de un discurso social elaborado sobre la relación géneropoder» (2007, p. 26). Como señalan Anguita y Torrego (2009), el reto sigue estando en ofrecer a los nuevos profesores y a las nuevas profesoras formación suficiente y de manera integral para que entiendan que el desafío de la igualdad es una necesidad social que no permite más aplazamientos en el campo educativo, ya que desde ese contexto podemos influir en las vidas y las visiones de las nuevas generaciones de forma definitiva.

Como comenta Díaz de Greñu (2010), aunque desde el profesorado se han planteado múltiples iniciativas y proyectos de innovación dónde se han contemplado la coeducación; estos no han tenido continuidad. Debido a las políticas educativas que se han llevado a cabo con respecto a la formación permanente del profesorado desde las administraciones educativas y el instituto de la mujer.

\section{Objetivos de la investigación}

Nos interesaba indagar si la formación inicial que se imparte al futuro profesorado de Educación Primaria les habilitaba en conocimientos, procedimientos y actitudes para ejercer competencias de género y trabajar de manera integral la coeducación. Por ello, planteamos diferentes objetivos que nos han guiado en los momentos de la investigación y a su vez, establecían la finalidad de esta.

Cohen \& Manion (1994), entienden que la investigación es un "procedimiento centrado en su objeto y pensado para tratar un problema concreto localizado en una situación inmediata» (p. 192). Se controlan ciertas variables mediante diferentes mecanismos que permitan ajustar el tema que se está investigando.

Antes de nada, estimamos conveniente aclarar que de acuerdo con Rodríguez y Valldeoriola (2009), la esencia de un trabajo de investigación se encuentra en el planteamiento de uno o varios objetivos, así como en la finalidad con la que se establecen. Entendemos por objetivo aquello que se 
pretende obtener con la elaboración de un proyecto. De esta forma, se orienta la investigación, por lo que, deben actuar en coherencia con la metodología.

El objetivo general de la presente investigación es el siguiente: Detectar el nivel de formación inicial en materia de coeducación del alumnado del Grado de Educación Primaria.

Además, derivados del objetivo general nos marcamos los siguientes objetivos específicos:

- Medir las competencias en materia de coeducación del alumnado del Grado de Educación Primaria.

- Comprobar si el alumnado del Grado de Educación Primaria reproduce estereotipos de género.

- Examinar la importancia de la coeducación en su rol como docentes del alumnado del Grado de Educación Primaria.

- Sondear los déficits del alumnado del Grado de Educación Primaria en materia de coeducación.

- Realizar propuestas de formación adaptados a las necesidades del alumnado en materia de coeducación.

- Concienciar sobre la importancia de la inclusión de la coeducación Educación Primaria.

\section{Metodología de la investigación}

De forma general, el método es la manera en la que el investigador o investigadora pretende solucionar una cuestión generando conocimiento. Como señala Muñoz, Rodríguez y Domínguez (2003), las actuaciones han de ser adecuadas al tipo de objeto estudiado y al problema planteado. A la hora de llevar a cabo una metodología se debe de definir, explicar y justificar el método elegido, ya que se pueden adoptar diferentes enfoques que definirán la naturaleza de la investigación.

Por lo tanto, desde una metodología cuantitativa llevamos a cabo una investigación objetiva, con la cual conocemos el comportamiento de una parte de la sociedad mediante un resultado que se encuadra en un momento determinado. Por lo que, esta investigación, eminentemente descriptiva, nos lleva a generar nuestro propio conocimiento acerca de la idea del alumnado de Educación Primaria de la Universidad de Cádiz sobre la coeducación.

Aunque es importante tener en cuenta que en la metodología cuantitativa existe un riesgo intrínseco a la generalización de la muestra, este se destruye cuando se realiza la investigación con una muestra lo suficientemente grande. Además, como bien señala Bell (2002), es importante que este proyecto nunca termine, es decir, que sea un ciclo que no concluya, que vaya mejorando mediante la constante revisión y evaluación, según las necesidades planteadas en cada momento.

\section{Metodología}

El instrumento elegido en la presente investigación es el cuestionario, el cual consiste en una secuencia de preguntas sobre aspectos de una investigación o evaluación. Se distinguen cuestionarios con preguntas cerradas y abiertas, dependiendo de la manera de la formulación.

Existen diferentes aspectos a tener en cuenta a la hora de construir un cuestionario como el orden de las preguntas, el diseño, la presentación o si son preguntas directas o indirectas, es decir, si el contenido objeto de la investigación está implícito o no en la respuesta de la persona entrevistada.

Todo ello repercute en las respuestas de los sujetos, así como en los resultados de la investigación. Según Fox (1981), la preparación y selección de los modelos que se utilizarán como respuesta significan la parte más importante de los cuestionarios.

Destacamos que las respuestas o categorías que se ofrezcan a este tipo de preguntas han de reunir dos condiciones esenciales: que sean exhaustivas y excluyentes. Entendemos como exhaustivas las respuestas o categorías que contemplan todas las opciones y casos que pueden darse. De esta forma el usuario que realiza las cuestiones no se plantea dejar de responder por no encontrar una respuesta adecuada. Además, son excluyentes cuando únicamente una de las alternativas es válida, así no se pueden seleccionar dos como correctas en la misma cuestión (Sierra, 1988).

Como señalan Rodríguez, Gil y García (1996, p. 1185):

«este instrumento se asocia a enfoques y diseños de investigación típicamente cuantitativos, porque se construye para contrastar puntos de vista, porque favorece el acercamiento a formas de conocimiento nomotético no ideográfico, porque su análisis se apoya en el uso de 
estadísticos que pretenden acercar los resultados en unos poco elementos (muestra) a un punto de referencia más amplio y definitorio (población) y en definitiva, porque suelen diseñarse y analizarse sin contar con otras perspectivas que aquélla que refleja el punto de vista del investigador.»

Al trabajar en clase con el alumnado del Grado de Educación Primaria el tema de la coeducación, se detectó un gran desconocimiento sobre el mismo. Además, a medida que se profundizó a través de entrevistas informales sobre el tema, el alumnado mostraba una gran confusión con los términos asociados a esta cuestión. Al seguir indagando sobre el tema se preguntó al profesorado, que imparte asignaturas relacionadas con la coeducación si en los nuevos planes de estudio, en el marco del Plan Bolonia, existían asignaturas que abordasen este asunto. El resultado de esta primera aproximación no formal fue la constatación de la total ausencia de tales cuestiones en las asignaturas obligatorias y optativas; tampoco se advertía alguna en las menciones del grado de Educación Primaria.

Por ello, para lograr la objetividad, conocer la opinión y el conocimiento general del alumnado realizamos un cuestionario cerrado con 10 preguntas. Se trata de un cuestionario anónimo realizado de manera presencial durante un tiempo estimado de 15 minutos al inicio de una lección docente. Además, previamente a la batería de preguntas incluimos tres cuestiones para conocer la edad, sexo y procedencia de los sujetos de la investigación. Para la validación del cuestionario, previamente a la presentación al alumnado, este cuestionario se le entregó a un comité de expertos que valoró si era adecuado e hizo un juicio crítico sobre tal. Cabero y Llorente (2013) comentan que los juicios emitidos por el comité de expertos suponen ventajas en la calidad del objeto de estudio en las respuestas planteadas. La comisión de especialistas valoró de manera positiva la implantación del cuestionario planteado ratificando su validación.

A continuación, presentamos el cuestionario utilizado en la presente investigación:

-¿Sabes que es la coeducación?

$\mathrm{Si} / \mathrm{No} /$ Tengo dudas

-¿Has recibido formación en coeducación a lo largo del Grado de Educación Primaria?

Ninguna / Poco / Bastante

- ¿Te ves capacitado/a profesionalmente para trabajar en un futuro la coeducación en un aula de Educación Primaria?

No, nada/ Poco/ Sí, lo estoy

-¿Crees que es necesario recibir formación sobre coeducación en el Grado de Educación Primaria? $\mathrm{Si} / \mathrm{No} / \mathrm{Me}$ es indiferente

-¿Consideras la coeducación un elemento importante en la educación de los niños y las niñas?

$\mathrm{Si} / \mathrm{No} / \mathrm{Me}$ es indiferente

-¿Cómo crees que debe de trabajarse la coeducación en el aula?

De manera puntual / Transversalmente / Integrado curricularmente

-¿Crees que la coeducación incide en la igualdad de oportunidades entre hombres y mujeres?

$\mathrm{Si} / \mathrm{No} / \mathrm{Algo}$

- ¿Crees que la coeducación que se lleva a cabo en las aulas de Educación Primaria actualmente es positiva?

Si / No / Puede mejorar

-¿Conoces materiales para trabajar la coeducación en el aula?

Bastantes / Algunos / Ninguno

-¿Crees que el profesorado tiene estereotipos de género que transmite al alumnado?

$\mathrm{Si} / \mathrm{No} /$ Algunos

\section{Muestra}

A la hora de llevar a cabo una investigación uno de los momentos fundamentales es la elección de la muestra, con ello nos referimos a la cantidad de usuarios que realizan el cuestionario. Para que la muestra sea representativa de una población debe ser lo suficientemente amplia y los usuarios deben de formar parte de la población de la cual deseamos conocer la información.

El cuestionario se realizó de forma anónima al alumnado de $4^{\circ}$ curso del Grado de Educación Primaria de la Facultad de Ciencias de la Educación de la Universidad de Cádiz; futuras maestras y 
futuros maestros, durante el curso académico 2017-2018. En total participaron 195 usuarios pertenecientes a tres grupos de 62, 64 y 69. El alumnado elegido ha realizado durante el segundo semestre del tercer curso del grado universitario y durante el primer semestre del cuarto curso prácticas en centros escolares. Por ello, esta muestra aporta información relevante sobre la realidad de la coeducación en las aulas, ya que se encuentra en contacto directo con el contexto educativo.

En el primer grupo, de 62 personas, encontramos 50 mujeres (80,65\%) y 12 hombres (19,35\%). La mayoría del alumnado tiene entre 22 y 27 años, aunque las edades están comprendidas entre 21 y 34 años.

En el segundo grupo, de 64 personas, encontramos 51 mujeres (79,70\%) y 13 hombres (20,30\%). La mayoría del alumnado tiene entre 21 y 26 años, aunque las edades están comprendidas entre 21 y 37 años.

Por último, en el tercer grupo hay 69 personas, de las cuales 54 son mujeres $(78,26 \%)$ y 15 son hombres $(21,74 \%)$.La mayoría del alumnado tiene entre 21 y 26 años, aunque las edades están comprendidas entre 21 y 40 años.

$\mathrm{Y}$ en general, en todos los grupos hay poca diversidad cultural, la gran mayoría del alumnado es español, exceptuando dos personas naturales de Senegal; ocho alumnos y alumnas Erasmus de Alemania, Italia y Finlandia y cuatro alumnas y alumnos de Méjico.

Esta actividad investigativa esclarece qué tipo de formación ha recibido el futuro profesorado de Educación Primaria durante su paso por la Universidad. Nos proporciona información acerca de la concepción del alumnado sobre la coeducación, así como nos permite vislumbrar de qué manera se llevará a cabo la coeducación por estos profesionales.

\section{Resultados}

Tras la realización por parte del alumnado de $4^{\circ}$ del Grado de Educación Primaria de los cuestionarios propuestos procedemos a analizar los datos de dicho instrumento de investigación.

Comenzando con la primera pregunta ¿Sabes que es la coeducación? Los datos obtenidos señalan que 93 saben qué es la coeducación, mientras que 81 tiene dudas y 21 no conocen este término.

Figura 1.

Representación de las respuestas a la pregunta ¿Sabes que es la coeducación?

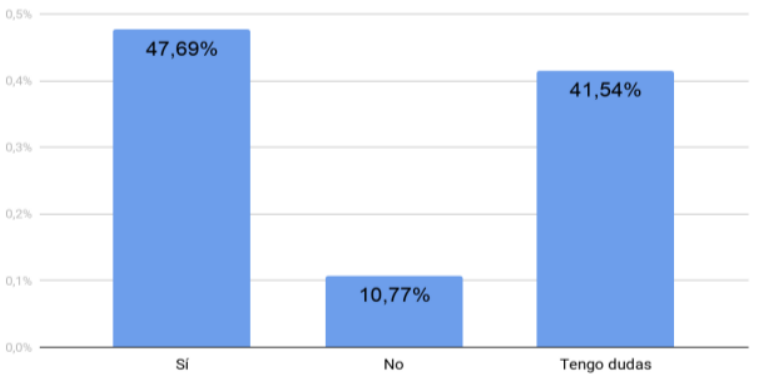

Fuente: Elaboración propia.

Con referencia a la siguiente pregunta ¿Has recibido formación en coeducación a lo largo de Educación Primaria? El alumnado apunta que 103 de ellos han recibido poca formación en materia de coeducación, mientras que 92 alumnos y alumnas señalan que no han recibido formación sobre esta temática. Resaltamos que ningún alumno señala que ha recibido bastante formación en la temática de estudio de la presente investigación.

Figura 2.

Representación de las respuestas a la pregunta ¿Has recibido formación en coeducación a lo largo del Grado de Educación Primaria?

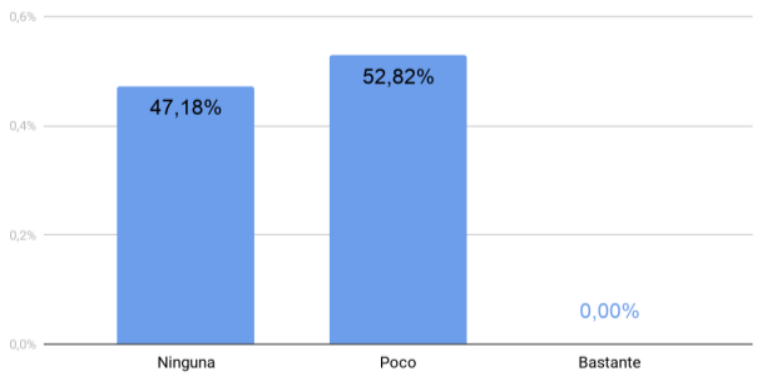

Fuente: Elaboración propia. 
Con respecto a la tercera pregunta ¿Te ves capacitado/a profesionalmente para trabajar en un futuro la coeducación en un aula de Educación Primaria? Concretamente, 63 alumnos y alumnas reconocen que no se encuentran preparados para llevar a cabo la coeducación en un futuro en sus centros escolares. Frente a 96 alumnos y alumnas que se sienten poco capacitados y 36 discentes que afirman tener competencias para trabajar esta materia en sus aulas.

Figura 3.

Representación de las respuestas a la pregunta ¿Te ves capacitado/ a profesionalmente para trabajar en un futuro la coeducación en un aula de Educación Primaria?

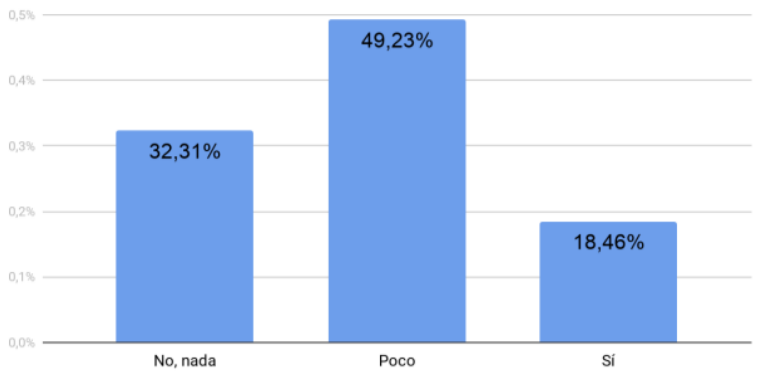

Fuente: Elaboración propia.

En la siguiente cuestión ¿Crees que es necesario recibir formación sobre coeducación en el grado de Educación Primaria? La mayoría del alumnado, exactamente 133 desean recibir formación en coeducación, frente a 39 alumnos y alumnas que no desea recibir formación en esta materia. Señalamos que 23 alumnos y alumnas comentan que les es indiferente.

Figura 4.

Representación de las respuestas a la pregunta ¿Crees que es necesario recibir formación sobre coeducación en el grado de Educación Primaria?

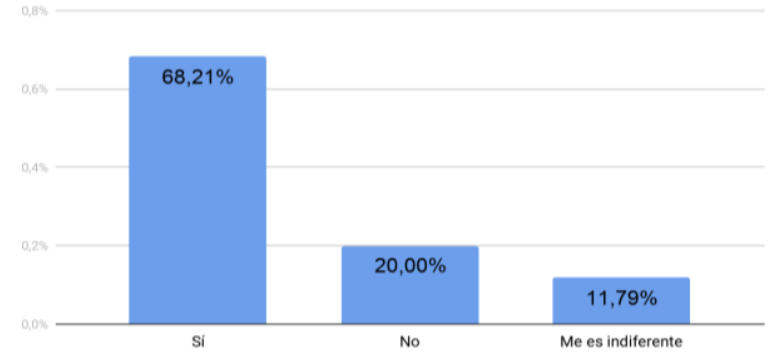

Fuente: Elaboración propia.

En relación a la pregunta ¿Consideras la coeducación un elemento importante en la educación de los niños y las niñas? Una mayoría de 158 alumnos y alumnas piensan que coeducación es un elemento importante en la educación del alumnado. Mientras, 29 alumnos y alumnas señalan que la coeducación no es importante, solo 8 alumnos y alumnas apuntan que les es indiferente.

Figura 5 .

Representación de las respuestas a la pregunta ¿Consideras la coeducación un elemento importante en la educación de los niños y las niñas?

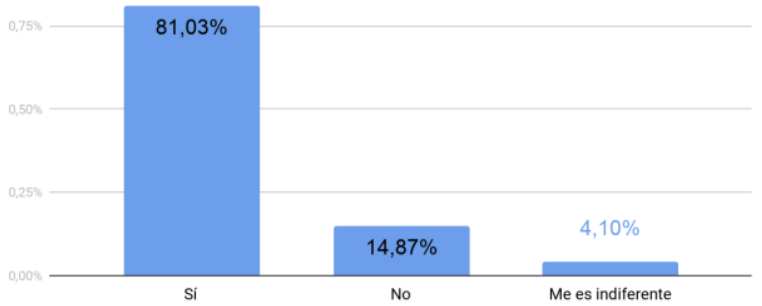

Fuente: Elaboración propia. 
Con respecto a la sexta pregunta ¿Cómo crees que debe de trabajarse la coeducación en el aula? Exactamente 95 alumnos y alumnas piensan que la coeducación se debe de trabajar integrada curricularmente, 63 alumnos y alumnas consideran que la educación se debe trabajar transversalmente y 37 señalan que debe de hacerse de manera puntual.

Figura 6.

Representación de las respuestas a la pregunta ¿Cómo crees que debe de trabajarse la coeducación en el aula?

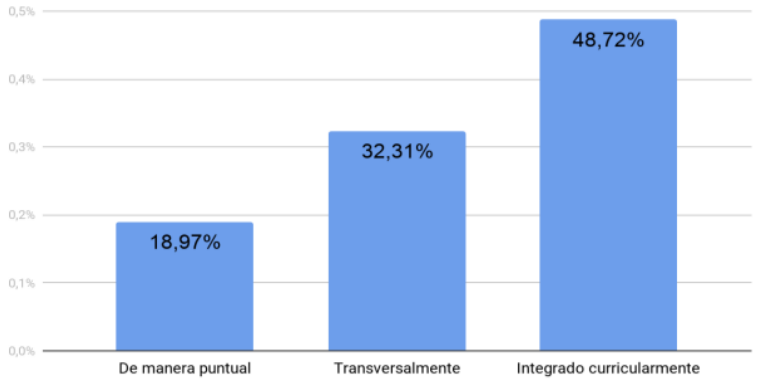

Fuente: Elaboración propia.

Continuando con la siguiente cuestión ¿Crees que la coeducación incide en la igualdad de oportunidades entre hombres y mujeres? Concretamente, 115 alumnos y alumnas consideran que la coeducación es un elemento que incide en la igualdad de oportunidades entre hombres y mujeres. Frente a 59 alumnos y alumnas que piensan que incide algo; mientras que 21 apuntan que la coeducación no incide en ello.

Figura 7.

Representación de las respuestas a la pregunta ¿Crees que la coeducación incide en la igualdad de oportunidades entre bombres y mijeres?

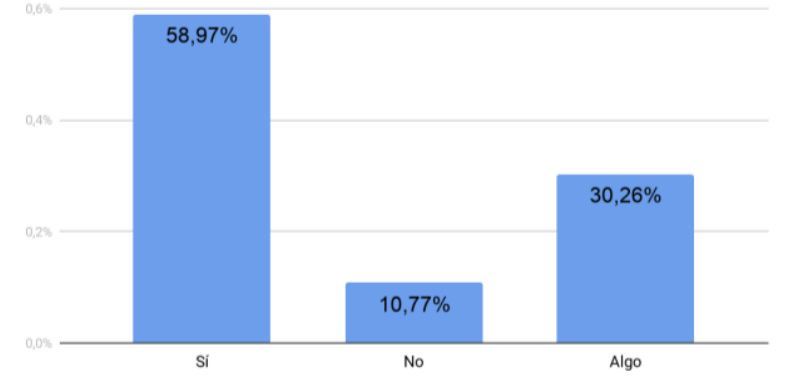

Fruente: Elaboración propia.

La siguiente cuestión ¿Crees que la coeducación que se lleva a cabo en las aulas de Educación Primaria actualmente es positiva? Nos revela que 84 alumnos y alumnas piensan que la coeducación que se trabaja actualmente puede mejorar. Frente a 71 alumnos y alumnas que opinan que la coeducación sí se está llevando a cabo de forma positiva. Concretamente, 40 alumnos y alumnas consideran que no se está llevando a cabo una coeducación de manera positiva.

Figura 8.

Representación de las respuestas a la pregunta ¿Crees que la coeducación que se lleva a cabo en las aulas de Educación Primaria actualmente es positiva?

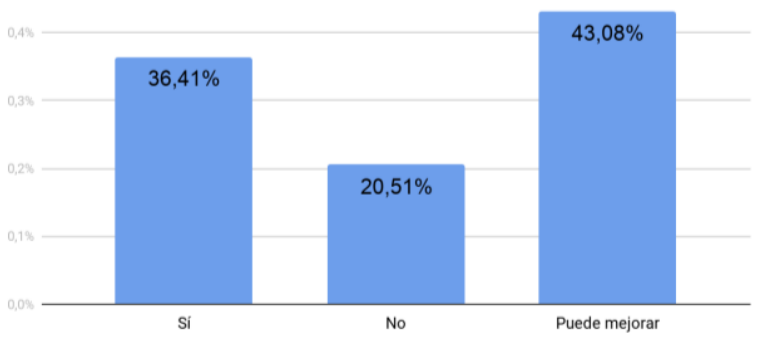

Fuente: Elaboración propia. 
A continuación, la pregunta ¿Conoces materiales para trabajar la coeducación en el aula? nos indica que 126 alumnos y alumnas no conocen materiales para trabajar la coeducación en sus futuras aulas. Mientras que 37 alumnos y alumnas afirman conocer bastantes materiales coeducativos y 32 algunos recursos para trabajar la coeducación.

Figura 9.

Representación de las respuestas a la pregunta ¿Conoces materiales para trabajar la coeducación en el aula?

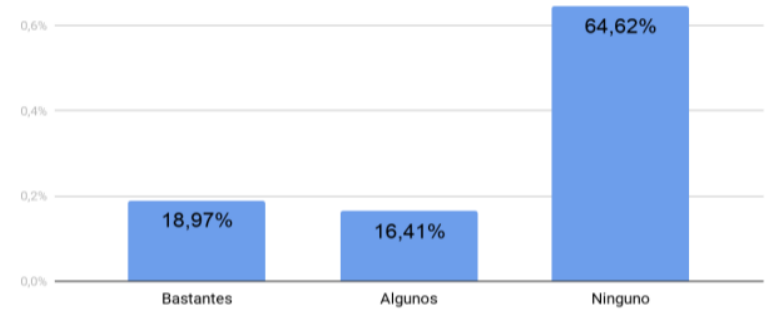

Fuente: Elaboración propia.

La última pregunta ¿Crees que el profesorado tiene estereotipos de género que transmite al alumnado? Nos muestra que 114 alumnos y alumnas afirman tener estereotipos sexistas. Con respecto a 47 alumnos y alumnas que señalan que tienen algunos estereotipos de género y 34 dicen que no tienen.

Figura 10.

Representación de las respuestas a la pregunta ¿Crees que el profesorado tiene estereotipos de género que transmite al alumnado?

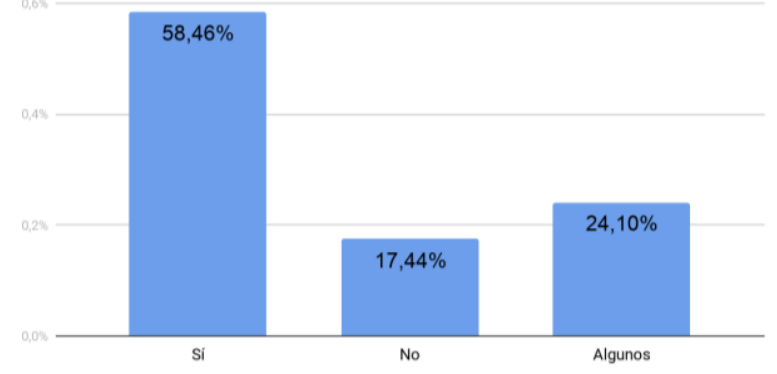

Fuente: Elaboración propia.

\section{Discusión y conclusiones}

Tras el análisis de los cuestionarios realizados llegamos a las reflexiones finales del estudio de la presente investigación. Hemos detectado que con respecto al concepto de coeducación gran parte del alumnado del Grado de Educación Primaria tiene dudas sobre este término y existe un 10 \% que no lo conoce en absoluto. Resaltamos que la mayoría de las personas que han realizado el cuestionario admiten que han recibido poca o ninguna formación en materia de coeducación. Hacemos hincapié en que ningún alumno ni alumna apuntan que han recibido los suficientes conocimientos en este ámbito. Se aprecia gran desconocimiento de este concepto y falta de formación. Esto nos lleva a pensar que la coeducación sigue siendo un tema que no se prioriza en los planes de formación inicial del profesorado provocando confusión e ignorancia en la materia.

Con respecto a la capacitación, la mayoría del alumnado expresa sentirse poco o nada competente para trabajar la coeducación en un futuro en sus aulas. Unido a la escasa formación anteriormente reflejada, detectamos que existen grandes obstáculos en la formación inicial del profesorado que impide la puesta en marcha de una escuela coeducativa.

Nos parece muy interesante, resaltar que la mayoría del alumnado desearía recibir formación en coeducación y el $80 \%$ lo considera importante en la educación de los niños y las niñas. Por lo que, observamos que el propio alumnado demanda la necesidad de formación en coeducación para construir una escuela libre de sexismo. Además, puntualizan que la mejor manera de trabajar la coeducación integrándola en el currículum y transversalmente. 
Un amplio número de alumnos y alumnas señalan la incidencia de la coeducación en la igualdad de oportunidades entre hombres y mujeres. Esto nos lleva a la reflexión de que el alumnado es consciente de la importancia de una escuela libre de sexismo en la construcción de una sociedad igualitaria. El propio alumnado estima que la coeducación que se lleva a cabo en los centros escolares puede mejorar.

Señalar que los y las discentes apuntan que no conocen materiales coeducativos con los que llevarían a cabo una práctica docente orientada a la igualdad entre niños y niñas. Para finalizar, un número considerable de alumnas y alumnos apuntan que poseen estereotipos de género. Este aspecto junto a la escasa formación que hemos detectado en la presente investigación nos lleva a plantearnos la urgente necesidad de una adecuada formación inicial del futuro profesorado en materia de coeducación.

No podemos olvidar la alta feminización de los estudios de formación de profesorado como señalan Barquín y Melero (1994), existe una mayor presencia de profesoras en el colectivo docente, que ha ido aumentando en los últimos años. Observamos claramente en la presente investigación el gran porcentaje de alumnas $(79,53 \%)$ frente a la representación de alumnos $(20,46 \%)$. Como apuntan Clandinin y Connelly (1987) las propias docentes perpetúan actitudes sexistas en su propia práctica educativa, ya que su propia biografía se ha desarrollado en una sociedad sexista y esto se refleja en su práctica docente.

Para finalizar, concluimos que esta investigación refleja, la necesidad de mejorar la formación inicial del futuro profesorado de Educación Primaria en materia de igualdad con el objeto de sensibilizar y aumentar el conocimiento de los docentes y de esta manera, como apuntan García-Perez, Vega, Barragán, Buzón y Piedra (2011), tratar de superar la «ceguera de género». Esta «ceguera» se soluciona poniéndose «las gafas de género» a través de una formación inicial innovadora y práctica del futuro profesorado de Educación Primaria en coeducación, y construir así una escuela igualitaria que traspase el rosa y azul.

Es esencial que el futuro profesorado de Educación Primaria adquiera responsabilidades en materia de género, para ello, desde las facultades de Ciencias de la Educación se les debe proporcionar una debida formación inicial que parta de dialogar con las futuras maestras y los futuros maestros, ya que serán los protagonistas que marcarán las necesidades que observan en su propia formación. Debemos favorecer un futuro profesorado comprometido con el ejercicio del magisterio para transformar y mejorar la calidad educativa a través de una formación integral con perspectiva de género.

La presente investigación demuestra que los temas de igualdad deben tener mayor legitimidad y visibilidad en la formación inicial del profesorado de Educación Primaria. Las instituciones educativas deben promover que los temas de género tengan carácter obligatorio para todo el alumnado y estén presentes de forma continuada a lo largo de toda su formación. No podemos limitar su tratamiento solamente en la realización de simples cursos o de charlas puntuales de sensibilización, que no consideramos que sean negativas, pero no lo suficiente para trabajar de manera integral la coeducación.

Hemos observado que es necesario ofrecer nuevas experiencias formativas con perspectiva de género al alumnado, así como generar espacios de diálogo y debate para deconstruir los prejuicios sexistas que poseen y favorecer la reflexión y revisión de su currículum oculto y futuras prácticas docentes con el fin de propiciar una formación igualitaria. García-Pérez et al. (2011), postulan que es un aspecto clave la sensibilización y la formación en género del profesorado en los procesos de cambio educativo en esta materia, elementos fundamentales para poder llegar a construir nuevas prácticas y conocimientos y hacer efectiva y real la igualdad entre hombres y mujeres en una sociedad libre de sexismo.

Defendemos que la coeducación es el camino hacia una escuela libre de sexismo y por ende en una sociedad igualitaria entre hombres y mujeres, detectamos la urgente necesidad de una estructura académica propicia a la reflexión con perspectiva de género, haciendo hincapié en los modelos implícitos desigualitarios, los estereotipos sexistas y el currículum oculto; para avanzar hacia una escuela libre de sexismo dónde la formación inicial del profesorado es un elemento clave.

Pérez Carracedo (2006), afirma el gran reto que tiene el sistema educativo para ofrecer a los nuevos profesores y a las nuevas profesoras una formación integral para que entiendan que el desafío de la igualdad es una necesidad social imperante en una sociedad democrática. Este reto no permite más aplazamientos en nuestras escuelas, ya que desde ese contexto podemos influir de manera igualitaria en las vidas y las visiones de las nuevas generaciones de forma definitiva en la construcción de una cultura de género. 


\section{Limitaciones del estudio}

Entre las diversas limitaciones del presente estudio podemos encontrar como señalan Prince \& Murnan (2004) la falta de estudios previos, ya que tras haber realizado una ardua revisión bibliográfica hemos detectado escasas investigaciones sobre la formación inicial del profesorado del Grado de Educación Primaria en materia de coeducación. Esta limitación supone una oportunidad para identificar nuevos nichos de investigación y nos plantea la urgente necesidad de seguir indagando sobre esta temática, para construir una escuela libre de sexismos.

Otra de las limitaciones que podemos observar es el sesgo de los datos auto-informados como señalan Prince \& Murnan (2004), entre las diferentes subjetividades de los y las informantes nos encontramos la memoria selectiva, obviar o rememorar sucesos que acontecieron, el «efecto telescopio», cuándo se recuerdan acontecimientos distorsionados en el tiempo, la atribución de eventos positivos a la persona informantes y hechos negativos a agentes externos y la exageración de la ponderación de los sucesos acaecidos.

Por último, desde el punto de vista de las limitaciones de las propias investigadoras señalamos el «efecto longitudinal», debido a la limitación de tiempo que se disponía para realizar la presente investigación no se han podido determinar cambios en el tiempo.

\section{Propuestas de intervención prácticas}

Nuestras propuestas demandan la capacidad del profesorado para interrogarse y cuestionar su práctica docente; es necesario que delibere sobre el campo epistémico desde donde construyen las diferencias de género. Por ello, abogamos el papel fundamental de la reflexión sobre la práctica docente como estrategia de mejora de la inclusión de la coeducación en los centros educativos. La reflexión de la práctica educativa es un proceso necesario para modificar y mejorar la práctica escolar. El profesorado debe cambiar las prácticas irreflexivas que provocan actuaciones sexistas y llegar a detectar las problemáticas y a su vez poder atenderlas con el propósito de buscar estrategias coeducativas.

Desde esta perspectiva, consideramos la reflexión como una experiencia necesaria, un medio y un método de repensar el modelo educativo sexista, por ello, es necesario reflexionar antes, durante y después de la acción educativa. Como postula Hagger (2006), es prioritario que el futuro profesorado teorice sobre su práctica educativa y, que reflexione sobre sus propias formas de actuar, sus limitaciones, sus creencias. El profesorado debe mantener un diálogo reflexivo con la práctica de aula y con las situaciones relacionadas con los roles sexistas.

La investigación es clave para mejorar y enriquecer el perfil profesional del profesorado. Apostar por docentes investigadores es apostar por profesionales con saberes y competencias desarrolladas a partir de la reflexión en su propia futura actuación docente. Tanto en los planes de formación inicial del profesorado, como en la oferta de formación permanente del profesorado en activo es una realidad que obstaculiza la formación en esta temática.

La formación del profesorado en materia de coeducación será útil para desarrollar una educación inclusiva de mayor calidad para todo el alumnado si se configura como un aspecto del sistema educativo que ayuda al cambio de la cultura profesional docente en un contexto abierto a todo el alumnado y orientado por valores igualitarios

Por todo esto, abogamos por una formación del profesorado que cuestione la visión sexista, que le ayude a romper el binarismo de género y la concepción estática de los roles de género.

Otro factor que consideramos necesario en la formación docente es la necesidad de formación permanente en materia de género, tal y como se expresa en La Ley Orgánica de Educación 2/2006, de 3 de mayo, modificada por la Ley para la Mejora de la Calidad Educativa 8/2013, de 9 de diciembre, donde en su artículo 102 establece que: «La formación permanente constituye un derecho y una obligación de todo el profesorado y una responsabilidad de las Administraciones educativas y de los propios centros».

Sería interesante plantear en el Máster de Formación de Profesorado de Educación Secundaria Obligatoria y Bachillerato, Formación Profesional y Enseñanzas de Idiomas, contenidos relacionados con esta cuestión. Proponemos en los centros permanentes de formación del profesorado, cursos, relacionados con la coeducación, para formar al personal docente en activo, y actualizar sus conocimientos y mejorando así su actuación docente. La creación de grupos permanentes de investigación para detectar las nuevas necesidades que vayan surgiendo en los centros educativos y plantear soluciones reales. 


\section{Referencias}

Altable, C. (1993). La coeducación sentimental. En J. Ramos (Coord.), El camino hacia una escuela coeducativa (pp. 129-144). Sevilla: M.C.E.P.

Anguita, R. \& Torrego, L. (2009). Género, educación y formación del profesorado. Retos y posibilidades. Revista Interuniversitaria de Formación del Profesorado, 64(23), pp. 17-25.

Antón, M. (Coord.). (2007). Planificar la etapa 0-6. Barcelona: Graó.

Ballarín, P. (2001). La educación de las mujeres en la España contemporánea. Madrid: Síntesis.

Barquín, J. \& Mejero, M. (1994). Feminización y profesión docente. Internalización sexista del trabajo. Investigación en la escuela, (22), pp. 25-34.

Bell, J. \& Filella, E. R. (2002). Cómo hacer tu primer trabajo de investigación: Guia para investigadores en educación y ciencias sociales. Barcelona: Gedisa Editorial.

Blant Gimeno, A. (1994). Informe sobre la igualdad de oportunidades educativas entre los sexos. Revista Iberoamericana de educación, pp. 1-18.

Blanco García, N. (2007). Coeducar es educar para la libertad. Andalucía educativa, (64), pp. 24-27.

Bolaños, L. M. (2007). Programa de formación del profesorado desde la perspectiva de género. $M a$ gistro, 1, pp. 25-34.

Bromme, R. (1988). Conocimientos profesionales de los profesores. Enseñanza de las Ciencias, 6(19), pp. 19-29.

Cabero Almenara, J. \& Llorente Cejudo, M. C. (2013). La aplicación del juicio de experto como técnica de evaluación de las tecnologías de la información (TIC). Eduweb. Revista de Tecnología de Información y Comunicación en Educación, 7(2), pp. 11-22.

Cabeza, A. (2010). Importancia de la coeducación en los centros educativos. Pedagogía Magna, (8), pp. 39-45.

Clandinin, J. \& Connelly, M. F. (1987). On narrative Method, Biography and Narrative Unities in the Study of Teaching. The Journal of Educational Thought. 21(3), pp.130-139.

Cohen, L. \& Manion, L. (1994). Research Methods in Education. Londres: Routledge.

Colás, P. (2004). La construcción de una pedagogía de género para la igualdad. En M. A. Rebollo \& I. Mercado (Coords.), Mujer y desarrollo en el siglo XXI: voces para la igualdad (pp. 275-291). Madrid: McGraw-Hill.

Díaz de Greñu, S. (2010). Discriminación o igualdad. La educación en el respeto a la diferencia a través de la enseñanza de la Historia. (Tesis Doctoral inédita). Valladolid, Universidad de Valladolid.

Escolano, A. (2002). La educación en la España contemporánea. Políticas educativas, escolarización y culturas pedagógicas. Madrid: Biblioteca Nueva.

Fernández, E. (1996). Representaciones diferenciadas sobre actividad física y género en el futuro profesorado de educación primaria. En F. del Villar (Coord.), La investigación en la enseñanza de la educación física. (p. 105). Madrid: Universidad de Extremadura. 
Flecha, C. (1989). Algunos aspectos sobre la mujer en la política educativa durante el régimen de Franco. Historia de la Educación, 8, pp. 77-97.

Fox, D. J. (1981). El proceso de investigación en Educación. Pamplona: Eunsa.

Freixas, A., Fuentes-Guerra, M., \& Luque, B. (2006). Formación del profesorado y diferencia sexual. Revista Fuentes, 7, pp. 52-64.

García-Pérez, R., Rebollo, M. A.; Vega, L.; Barragán, R.; Buzón, O., \& Piedra, J. (2011). El Patriarcado no es Transparente: Competencias del Profesorado para Reconocer la Desigualdad. Culturay Educación, 23(3), pp. 385-397.

García, R., Sala, A., Rodríguez, E., \& Sabuco, A. (2013). Formación inicial del profesorado sobre género y coeducación: impactos metacognitivos de la inclusión curricular transversal sobre sexismo y homofobia. Profesorado. Revista de currículum y formación del profesorado, 17(1), pp. 269-287. Recuperado de: http://www.ugr.es/ recfpro/rev171COL1.pdf.

Hagger, H. \& Mcintyre, D. (2006). Learning teaching from teachers. New York: Open University Press:.

Instituto de la mujer/red2 consultores (2004). Estudio para conocer la situación actual de la educación para la igualdad en España. Madrid: Instituto de la Mujer.

Ley de Instrucción pública de 1857.

Ley 14/1970, de 4 de agosto, General de Educación y Financiamiento de la Reforma Educativa. Boletín Oficial del Estado.

Ley Orgánica General del Sistema Educativo, número 238 de 3 de octubre de 1990. Boletín Oficial del Estado.

Ley Orgánica 2/2006, número 106 de 3 de mayo de Educación. Boletín Oficial del Estado.

Ley Orgánica 3/2007, de 22 de marzo, para la igualdad efectiva de mujeres y hombres. Boletín Oficial del Estado.

Ley Orgánica 8/2013, número 295 de 9 de diciembre del 2016, para la mejora de la calidad educativa. Boletín Oficial del Estado.

Martín Vico, M. J. (2007). Género y formación del profesorado. Consejería de Educación. Andalucía educativa, 64, pp. 31-33.

Monasterio, M., González, S., \& García, A. (2011) La coeducación en la escuela del siglo XXI. Madrid: Catarata.

Pérez Carracedo, L. (12 de enero de 2006). Por la plena integración de los estudios de las mujeres, feministas y de género en el espacio europeo de educación superior. El periódico feminista. Recuperado de: http://www.mujeresenred.net/spip.php?article459.

Price, J.H. \& Murnan, J. (2004). Research Limitations and the Necessity of Reporting Them. American Journal of Health Education, 35, pp. 66-67.

Puelles, M. (1991). Educación e ideología en la España contemporánea. Barcelona: Labor.

Real Decreto 861/2010, de 2 de julio, por el que se modifica el Real Decreto 1393/2007, de 29 de octubre, por el que se establece la ordenación de las enseñanzas universitarias oficiales. 
Rebollo, M. A. (2013). La innovación educativa con perspectiva de género. Retos y desafíos para el profesorado. Revista de currículum y formación del profesorado, 17(3), pp. 3-8. Recuperado de: www.ugr.es/ recfpro/rev171ed.pdf .

Rodríguez, D. \& Valldeoriola, J. (2009). Metodología de la investigación. Barcelona: Universitat Oberta de Catalunya. Recuperado de: http://myuvmcollege.com/uploads/lectura201109/Metodo$\log \% \mathrm{C} 3 \% \mathrm{ADa} \% 20 \mathrm{de} \% 20$ investigaci $\% \mathrm{C} 3 \% \mathrm{~B} 3$ n-2064.pdf.

Rodríguez, G., Gil, J., \& García, E. (1996). Métodos de investigación cualitativa. Málaga: Aljibe.

Rodríguez Martínez, C., \& Dolz L. (2016). Los contenidos escolares y la metodología docente. En A. Garzón \& E. Díez (Coords.), La educación que necesitamos (pp. 56-65). Madrid: Foca.

Santos Guerra, M. A. (2008). La pedagogía contra Frankenstein: Y otros textos frente al desaliento educativo. Barcelona: Editorial Graó.

Scalon, G. (1986). La polémica feminista en la España contemporánea, 1868-1974. Madrid: Akal.

Scraton, S. (1992). Shaping up to womanhood: gender and girls' physical education. Buckingham: Open University Press.

Sierra Bravo, R. (1988). Técnicas de investigación Social. Teoría y Ejercicios. Madrid: Paraninfo.

Simón, E. (2000). Tiempos y espacios para la coeducación. En M. A. Santos Guerra (Coord.), El harén pedagógico. Perspectiva de género en la organización escolar. Barcelona: Graó.

Sohn, A. M. (2002). Los roles sexuales en Francia y en Inglaterra: una transición suave. En G. Duby \& M. Perrot (Coord.), Historia de las Mujeres. El siglo XX (pp.127-157). Madrid: Taurus.

Suberviola Ovejas, I. (2012). Coeducación: un derecho y un deber del profesorado. REIFOP, 15(3), pp. 59-67.

Subirats, M. \& Brullet, C. (1988). Rosa y Azul. Madrid: Ministerio de Cultura-Instituto de la Mujer.

Subirats, M. (1994). Conquistar la igualdad: la coeducación hoy. Revista Iberoamericana de Educación. Género y Educación, 6, pp. 1-26.

Webber, M. (2005). Don't be so feminist: Exploring student resistance to feminist approaches in a Canadian university. Women's Studies International Forum, 28, pp. 181-194.

Weiner, G. (2000). A critical review of gender and teacher education in Europe. Pedagogy, Culture and Society, 8, pp. 233-247. 